\title{
Enhanced Techniques for Asymmetry Quantification in Brain Imagery
}

\author{
Xin Liu $^{1}$, Celina Imielinska ${ }^{1,2}$, Joel Rosiene ${ }^{4}$, E. Sander Connolly ${ }^{4}$, Anthony L. D'Ambrosio ${ }^{4}$ \\ ${ }^{1}$ Department of Biomedical Informatics, Columbia University, New York, NY \\ ${ }^{2}$ Department of Computer Science, Columbia University, New York, NY \\ ${ }^{3}$ Department of Neurological Surgery, Columbia University, New York, NY \\ ${ }^{4}$ Department of Mathematics and Computer Science, Eastern Connecticut State University, CT
}

\begin{abstract}
We present an automated generic methodology for symmetry identification and asymmetry quantification, novel method of identifying and delineation of brain pathology by analyzing the opposing sides of the brain utilizing of inherent leftright symmetry in the brain. After symmetry axis has been detected, we apply non-parametric statistical tests operating on the pairs of samples to identify initial seeds points which is defined defined as the pixels where the most statistically significant difference appears. Local region growing is performed on the difference map, from where the seeds are aggregating until it captures all 8-way connected high signals from the difference map. We illustrate the capability of our method with examples ranging from tumors in patient MR data to animal stroke data. The validation results on Rat stroke data have shown that this approach has promise to achieve high precision and full automation in segmenting lesions in reflectional symmetrical objects.
\end{abstract}

Keywords: magnetic resonance, brain imaging, symmetry, computational geometry, non parametric test

\section{INTRODUCTION}

Medical image segmentation, that is a process of identifying and delineating anatomical structures and other objects in images, still largely remains an open problem, in spite of several decades of research from various imaging modalities [19]. There are many brain segmentation approaches evolved from low level image operation such as thresholding, edge detection, mathematical morphology[10], to more sophisticated image processing methods such as statistical classification[11][12], active contours[20], neural networks[4], fuzzy connectedness [21], and hybrid segmentation methods [22]. However, clinical image analysis indicates that to successfully differentiate between organ and tumor tissue image information alone is insufficient [6]. For example, if a tumor shows insufficient contrast against the healthy brain tissue, the active contour classification requires a selection of seed to initialize segmentation, hence the method is not fully automated. Other statistical classification methods are also limited due to overlapping intensity distributions of healthy tissue, tumor, and surrounding edema.

In the digital anatomical atlas -based segmentation [13] prior knowledge about normal brain anatomy is used, including the size, shape and location of anatomical structures. However, the shape and other characteristics of tumors and other brain pathologies are highly variable, thus representing prior knowledge is not always possible. In [6], Kaus et al propose an adaptive template moderated classification (ATMC) method that combines the statistical classification with anatomical knowledge. The algorithm involves iterative process of classification of patient's data and nonlinear registration to match the anatomical templates of a digital atlas with the brain anatomy of the patient. However, such atlases, obtained from manually segmented MR imaging are not always available and such an approach has a limited use.

In this paper, we propose a novel method of identifying and delineation of brain pathology by analyzing the opposing sides of the brain. In another words, we replace the need for a digital atlas with the cross-registration between opposing hemispheres of the brain. Because brain exhibits high level of bilateral symmetry and this symmetry is distorted in a

Medical Imaging 2006: Image Processing, edited by Joseph M. Reinhardt, Josien P. W. Pluim,

Proc. of SPIE Vol. 6144, 61446W, (2006) - 0277-786X/06/\$15 - doi: 10.1117/12.654063 
presence of a pathology, we utilize the inherent left-right symmetry in the brain and use the healthy side of the brain as our prior template to statistically enhance the differences in high dimensional feature space.

With our fully automated system we segment brain abnormalities such as tumors, stroke, and other pathologies. We replace spatial prior of a generic statistical normal human brain atlas with the subject-specific left-to-right symmetry information derived from the subject's brain images. We use human and rat data with stroke and tumors to demonstrate the capabilities of our method. We define a pipeline that can accurately capture the asymmetries between two hemispheres, quantify the discrepancies and segment the pathologies. In the Results section, we show examples that illustrate the pipeline.

We illustrate the capability of our method with examples ranging from tumors in patient MR data to animal stroke data. This method can be used to improve segmentation of pathology in structural brain images and as quantification of perfusion-weighted tomography. There in a number of clinical applications in neuro-radiology where this method can be useful: identification and segmentation of ischemic stroke in animal [1,23] and human models, quantification of magnetic resonance perfusion (MRP) changes in patients with cognitive deficits following carotid endarterectomy [2], objective quantification of perfusion-weighted computer tomography in the setting of acute aneurysmal subarachnoid hemorrhage [3,4].

The paper is organized as follows. In section 2, we will have a detailed description of our proposed algorithm. The results is presented in Section 3. We evaluate the results in Section 4 and conclude our work in Section V.

\section{METHODS}

We propose an automated segmentation tool, depicted in the flowchart in Fig. 1. that utilizes the inherent left-right symmetry in the brain. In the first step the symmetry axis is computed and the tilt of the head is corrected by applying standard affine transform. The detection of slight variations in the left to right brain imagery is complicated by normal differences in the anatomical structures occurring in the data set. Therefore, we utilize the apparent bi-lateral symmetry in brain imagery through the application of non-parametric statistical tests operating on the pairs of samples and nonparametric statistical tests on local averages. This technique can highlight regions that can be further examined in a "statistical" sense. The highlighted region can be used as "seeds" for later propagation in the difference map that quantifies differences between brain hemispheres $[3,4]$.

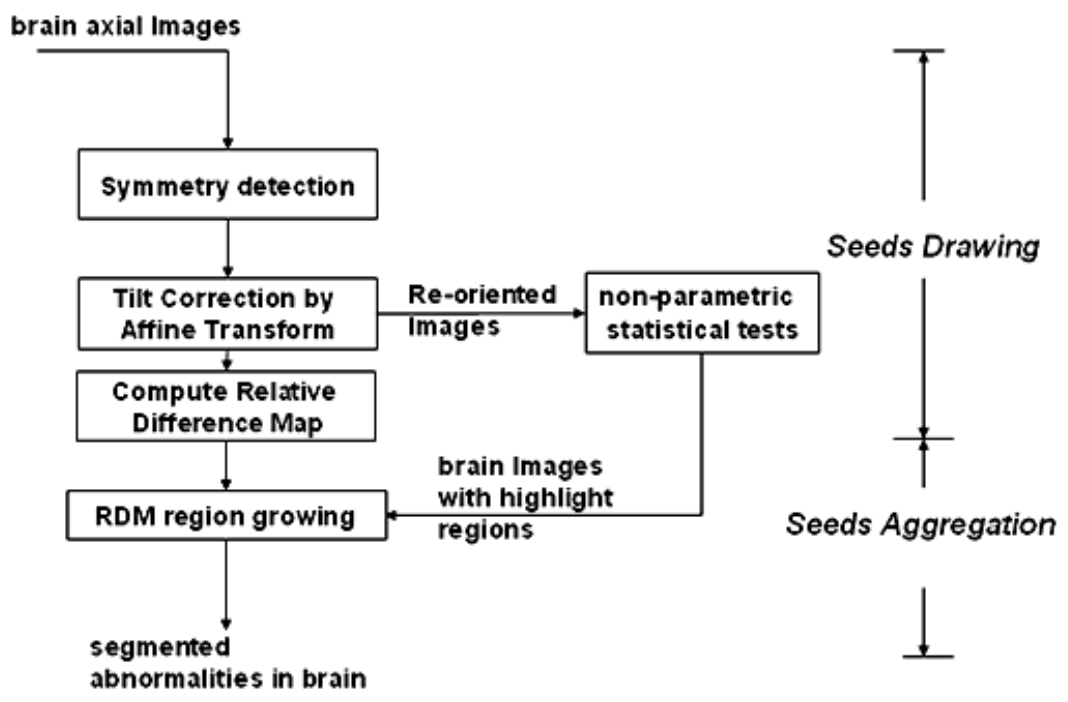

Fig.1 Flow chart of proposed segmentation scheme 
The statistical tests provide the "likelihood" of the difference to appear randomly given the samples, that has $1: \mathrm{N}$ chance of occurrence without un underlying difference being present. Finally a region is grown within the difference map yielding a segmentation of an abnormal target in the brain.

As shown in Fig.1, the major components of our proposed segmentation pipeline, consists of two consecutive steps, seeds drawing and seeds aggregation. Unlike most current region based segmentation approaches, seeds placement is fully automated. Seeds are understood as the occurrence of the regions with statistically minimal chances of an underlying symmetry being present.

In radiological brain images, to measure differences in bi-fold mirror symmetry the axis of symmetry must be computed and the image "self-registered" to this axis. After detection and registration of the image to the axis of symmetry, the data can be checked for significant differences in the image in regions collocated relative to the given axis of symmetry. This approach defines the essence of the relative difference map (RDM) method [3,4], that quantifies and highlights asymmetric areas in two brain hemispheres. In this paper, we refine the method of selecting an axis of symmetry and computation of relative asymmetry with respect to the axis of symmetry. Our method allows to compute the RDM for an image. We select a pair of symmetric windows (image elements), being ordered sets of points, about the axis of symmetry and compute set of relative differences with the associated statistics while scanning simultaneously both hemispheres.

Thus the seeds placement is further composed of the following sub-components: Symmetry detection, tilt correction and asymmetry measurement by non-parametric statistical tests.

\subsection{Symmetry Detection and asymmetry measurement-Seeds drawing}

Many clinical images are misaligned, tilted, a phenomenon that may be caused by the immobility of the patient, inexperience of the technician, or the imaging device itself, symmetry detection and correction of the misalignment comprises the first entry of the pipeline. Various approaches to detecting, analyzing, measuring and applying symmetry in image analysis have been suggested, see $[7,8,9]$. Some of them $[17,18]$ can even detect 3D symmetry out of the stack of images. In our early work[2-3], an algorithm for detecting the symmetry axes of a 2D planar shape is presented.

We summarize the seeds drawing algorithm into the following steps: 1) Identifying the axis of symmetry; Step 2) Affine transform to re-center and re-orient the image 3) Conducting statistical symmetry measurement to highlight the "sufficient" asymmetry;

\subsubsection{Identification of Symmetry Axis}

Let us assume that we have a single object in of interest. The area of the region $R=\{(x, y) \in R: I(x, y)>\alpha, \alpha>0\}$, with the given the characteristic function can be found as follows:

$$
A=\iint_{I} \chi(x, y) d x d x=\iint_{R} d x d y
$$

With the integration over the whole image I, and A defined as the $0^{\text {th }}$ moment of $\chi_{R}$ Then the center of the object is computed, that is that point where all the mass of the object could be concentrated without changing the first moment about any $\mathrm{x}$-axis[14].

$$
\begin{aligned}
& \bar{x}=\left(\iint_{R} x d x d y\right) / A \\
& \bar{y}=\left(\iint_{R} y d x d y\right) / A
\end{aligned}
$$

where $(\bar{x}, \bar{y})$ is the position of the center of area.

The technique follows rays emanating from the centroid $(\bar{x}, \bar{y})$ to the boundary elements of $\chi_{R}$. The intersections of the ray with the boundary of the region are recorded. Obviously, when the centroid is interior to the region of interest 
(ROI) all of the points on the boundary are path connected to centroid, and a ray extending from the centroid (a straight path) will intersect at least one point on the boundary [15].

The rays are parameterized by the discrete approximation to $\theta=k \Delta \theta, k \in Z$. (We will use $\theta$ that is discrete to simplify notation). The value of $\Delta \theta$ is chosen as in Fig.2 to insure that the step doesn't exceed $\sqrt{2}$ at a given maximum radius. For example, by selecting the points furthest away from the centroid this constructs the star shaped object, with each of the rays indexed by the angle. Any image $I(x, y) \leftrightarrow I(r, \theta)$ where the origin $(0,0)$ is in the centroid of the image, with the support of the function being $\chi_{R}(x, y)$. For an object with a well-defined boundary, there are lines $\mathbf{I}_{k}$ extending from the centroid $(\bar{x}, \bar{y})$ of length and direction $(r(\theta), \theta)$. In the polar representation, to determine the $k$ - fold mirror symmetric axes we seek the $\mathrm{K}$ values $\left\{\phi_{1}, \phi_{2}, \phi_{3}, \cdots \phi_{k}\right\}$ minimize the distance (in general)

$$
\begin{aligned}
& d_{\phi}^{p}(\text { Right }, \text { Left })=\sqrt[p]{\int_{\theta=0}^{\pi}|r(\phi+\theta)-r(\phi-\theta)|^{p} d \theta} \\
& \text { Specifically, for } \mathrm{p}=1, \\
& d_{\phi}^{1}(\text { Right }, \text { Left })=\int_{\theta=0}^{\pi}|r(\phi+\theta)-r(\phi-\theta)| d \theta
\end{aligned}
$$

with the best symmetry axis being

$$
\phi_{\tau}=\arg \min d_{\phi}^{1}(\text { Right, Left })
$$

the resulting $\phi_{\tau}$ that minimizes the distance are the minimum torque arms. Therefore, the problem of identifying symmetric axes is simplified to spot the global minimum of symmetry measure $\mathrm{S}$ in $\mathrm{r}-\theta$ space. This can be implemented as comparing the left half and right half of a sliding window across the entire $\mathrm{r}-\theta$ space, as shown in 2 . The window width is equivalent to the total amount of rays completing the transverse of polar space $(2 \pi)$ and the window height is equivalent to the maximum radius preset in the application. Therefore, we are essentially seeking such a state of equilibrium where the sum of all the difference between the left pixel and right counterpart over the space $\pi$, is the minimum (ideally zero), see Fig 2.
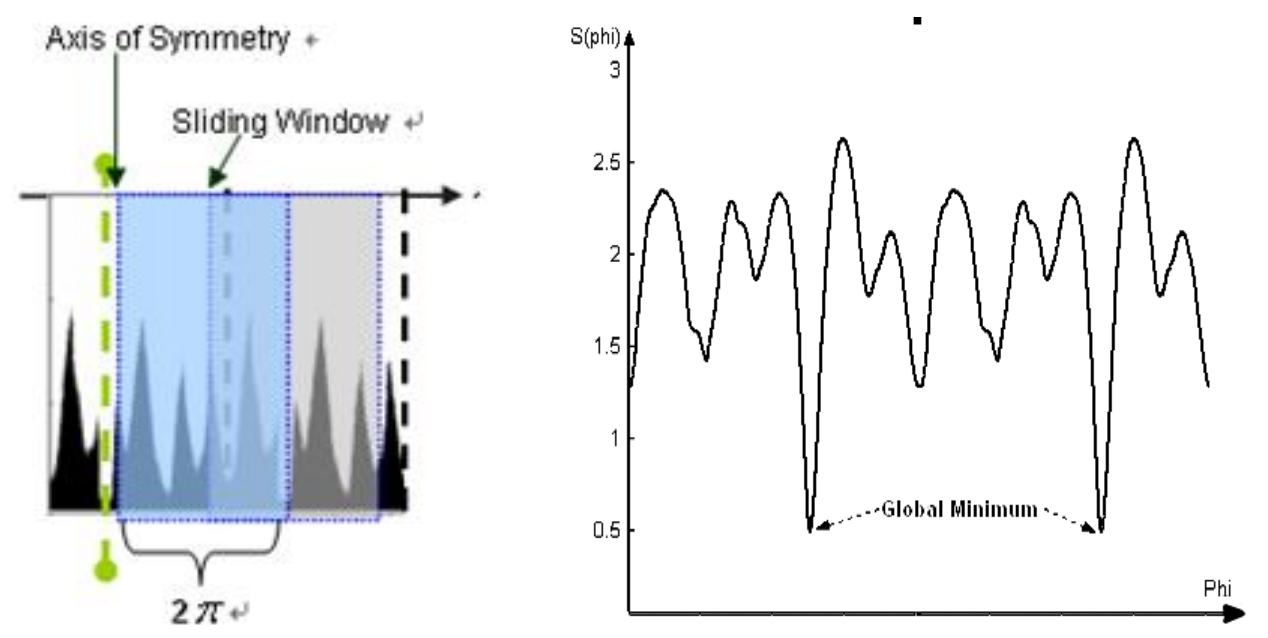

(a)

(b)

Fig. 2. (a)The axis of symmetry in $\mathrm{r}-\theta$ space. The sliding window has a width of $2 \pi$, and the minimal difference between two halves means that window traced through the object from one end of symmetry axes to another. (b) Symmetry measure $S(\Phi)$ along the $2 \pi$ space orientation. The global minimum of $\mathrm{S}$, indicates the orientation of symmetry axes. 


\subsubsection{Affine transform to re-center and re-orient the image}

Suppose that an image, $\mathrm{f}$, defined over a $(\mathrm{w}, \mathrm{z})$ coordinate system, undergoes geometric transformation to produce an image, g, defined over an $(\mathrm{x}, \mathrm{y})$ coordinate system. This transformation can be expressed as $[\mathrm{x} \mathrm{y} 1]=[\mathrm{w} \mathrm{z} 1] \mathrm{T}$, where $\mathrm{T}$ is called transformation matrix.

$$
T=\left[\begin{array}{ccc}
\cos \theta & \sin \theta & 0 \\
-\sin \theta & \cos \theta & 0 \\
\delta_{x} & \delta_{y} & 0
\end{array}\right]
$$

$\theta$ is the rotation angle, and $\left(\delta_{x}, \delta_{y}\right)$ is the translation offset between centroid of the brain and the center of the image.

\subsubsection{Application of Statistical Tests Highlight Asymmetry}

This method allows to compute the RDM for an image. We select a pair of symmetric windows (image elements), being ordered sets of points, about the axis of symmetry and compute set of relative differences with the associated statistics while scanning simultaneously both hemispheres. To create pairs of image elements the right or left set of pixels is flipped left to right (e.g., $\vec{X}_{R}$ ) allowing us to group pixels into regions that should be significantly similar. The neighborhood of a pixel is defined as a set of offsets from a row of columns, as in Equation 1. Equation 2. The neighborhood is used to create the set of image values about

$$
\begin{gathered}
N=\left\{\left(\Delta r_{0}, \Delta c_{0}\right),\left(\Delta r_{1}, \Delta c_{1}\right), \cdots\left(\Delta r_{k}, \Delta c_{k}\right)\right\} \\
S_{N}: X(r, c)=\left\{X\left(r^{\prime}, c^{\prime}\right):(r+\Delta r, c+\Delta c),(\Delta r, \Delta c) \in N\right\}
\end{gathered}
$$

the point $(r, c)$. Of course boundary and background points should be identified and treated appropriately. Secondly we use the Wilcoxon rank sum test for equal median on the elements paired elements and the $p$ value is recorded as in Equation 3.

$$
p(r, c)=\operatorname{ranksum}\left(\left\{S_{N}: X_{L}(r, c), S_{N}: \vec{X}_{R}(r, c)\right\}\right)
$$

The Wilcoxon rank sum test was selected since it compares the median of the pair wise differences of the two data sets with out the restrictions present in the Student's t-test, but other statistical tests can be substituted. In the form of the test $p$ is the probability of observing at least the statistic by chance if the medians are equal. The calculated probabilities can be used to estimate regions in the image that exhibit significant difference by applying a threshold as in Equation 4 to obtain a characteristic function.

$$
\chi_{\alpha}(r, c)=\left\{\begin{array}{l}
1, \forall(r, c): p(r, c)<\alpha, \alpha>0 \\
0, \text { otherwise }
\end{array}\right.
$$

Due to the natural variation present in the biomedical imagery it is possible that there neighborhood which minimizes the $p$ of the rank-sum test is slightly offset from the point of bi-fold symmetry. To compensate for this, the technique can consider a set of offsets as in Equation 5 that are used to shift the one of the neighborhoods to compensate for asymmetries and accumulate the $p$ values for all offsets to obtain an average

$$
O=\left\{\left(\Delta r_{0}^{\prime}, \Delta c^{\prime}{ }_{0}\right),\left(\Delta r_{1}^{\prime}, \Delta c_{1}^{\prime}\right) \cdots\left(\Delta r_{l}^{\prime}, \Delta c^{\prime}{ }_{l}\right)\right\}
$$

$p$ value as in Equation 6. 


$$
\bar{p}(r, c)=\frac{1}{|O|} \sum_{(\Delta r, \Delta c) \in O} \operatorname{ranksum}\left(\left\{S_{N}: X_{L}(r, c), S_{N}: \vec{X}_{R}(r+\Delta r, c+\Delta c)\right\}\right)
$$

the point $(\mathrm{r}, \mathrm{c})$. The boundary and background points should be identified and treated appropriately. Secondly we use the Wilcoxon rank sum test for equal median on the paired elements and the p value is recorded as in Equation 6.

\subsection{Region growing within the difference map---Seeds Aggregation}

The basic idea behind region growing is aggregating pixels (voxels) that are adjacent and belong to the same tissue type with fairly homogeneous grayscale properties[16]. Region growing approach consists of two major steps: first, selection of a set of seed points; second, growing of the region by appending the neighboring pixels (voxels) that have satisfied similarity criteria. In our segmentation pipeline, the seeds have been drawn from the preceeding step where the "seeds" can be defined as the pixels where the most statistically significant difference appears. Hence we provide a full automation of seed selection.

The stopping criteria of region growing is formulated based on intensity values of difference map. Since the "seed" assumes a certain number of points, we can caculate its mean and variance. Therefore, the value of each candidate pixel is compared with the average intensity of the seed region. A threshold value is used to test if the candidate is sufficiently similar to the seeds. In addition, if the pixel in question is 8 way connected to one or more seed values, then the pixel is considered a member of one or more regions. The region is being grown within difference map and a threshold value has been determined based on the variance of the region of interest.

The output of region that we grow with our methos is a labeled region (volume), where the label indicates the membership of a pixel (voxel) in a segmented object. A label of zero indicates that the voxel was not assigned to any object. In cases where brain has multiple lesions, the segmented image has multiple distinctive non-zero labels. For example, in Fig.6, the brain has two stroke areas therefore, it is being marked with two labels. Therefore, the proposed segmentation pipeline does not require a user to select an initial seed and the segmentation is fully automated.

\section{RESULTS}

We illustrate below our technique on a number of clinical and animal examples. Computation of relative asymmetric regions is used for quantification of perfusion-weighted images, and pre-segmentation of brain tumors from structural magnetic resonance imaging. The relative or original values are shown superimposed on the detected area of asymmetry. In quantification of perfusion-weighted images the relative values of perfusion parameter are displayed and highlighted in the detected area of asymmetry, Fig.3(b). In segmentation of brain tumor from structural MR data, Fig.5 and Fig.7, the tilt of head has firstly been corrected before the marking of the most significant difference.

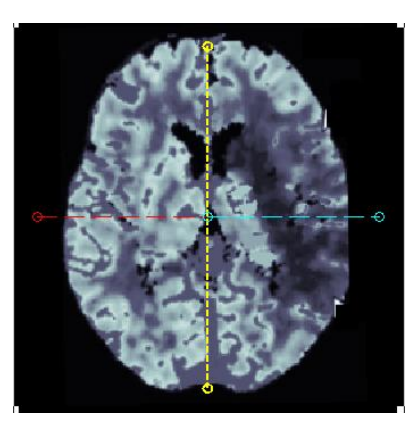

(a)

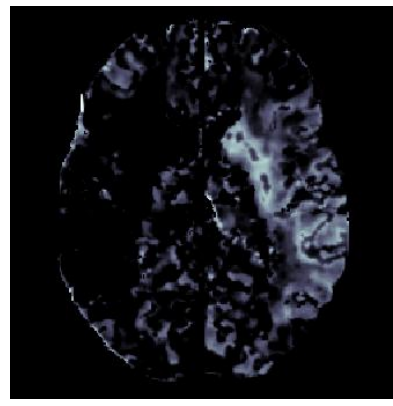

(b)

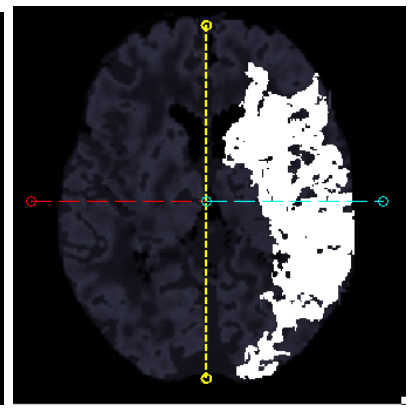

(c)

Fig.3 Perfusion-weighted computed tomography with ischemic stroke, consistent with left Middle Cerebral artery MCA infarction (This data was provided by Dr. Bernd Tomandl from Dept. of Neuroradiology at Univesrity of Erlangen-Nuremberg): (a) input data with a computed axis of symmetry, (b) relative difference map with relative cerebral blood flow (CBF) values in the area of detected asymmetry, (c) the statistical significant difference map. 

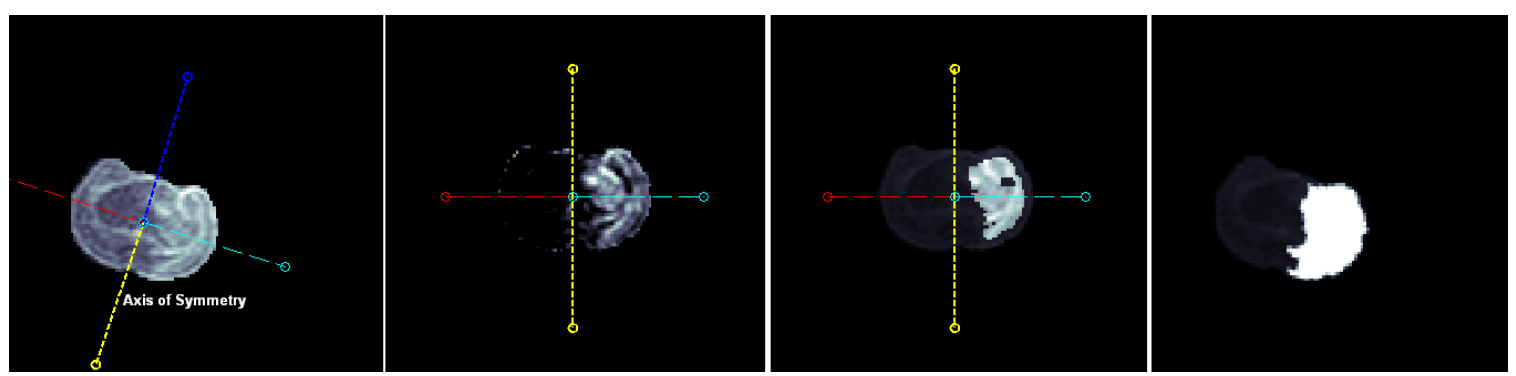

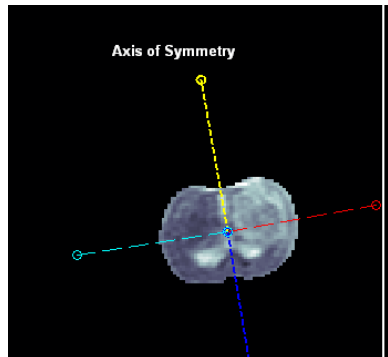

(a)

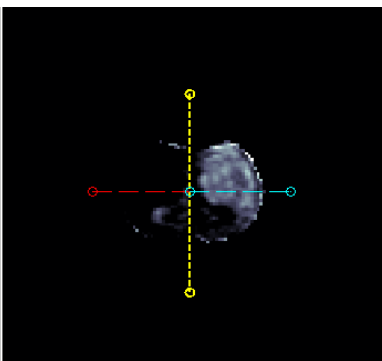

(b)

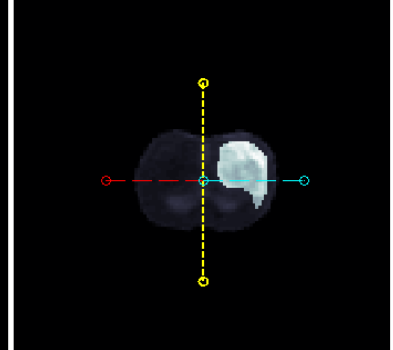

(c)

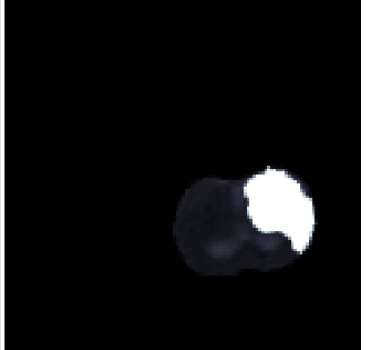

(d)

Fig.4. Rat Stroke Segmentation This data was provided by Dr. Anthony L. D'Ambrosio from Dept. of Neurosurgery at Columbia University Presbyterian Hospital. (a) are the original images;(b) are the difference map with corrected orientation;(c) are the statistical significant difference images with $\mathrm{p}$ value smaller than $5.0770 \mathrm{e}-014 ;(\mathrm{d})$ the final segmented images with original orientation.

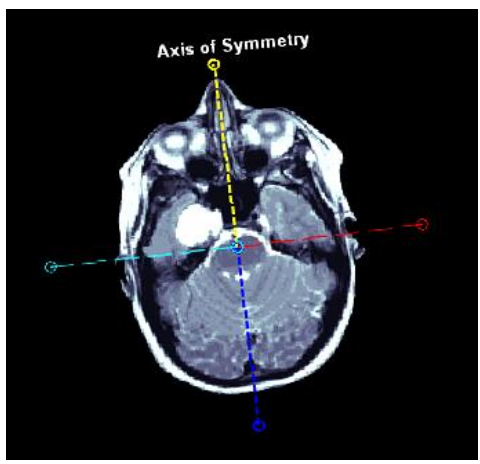

(a)

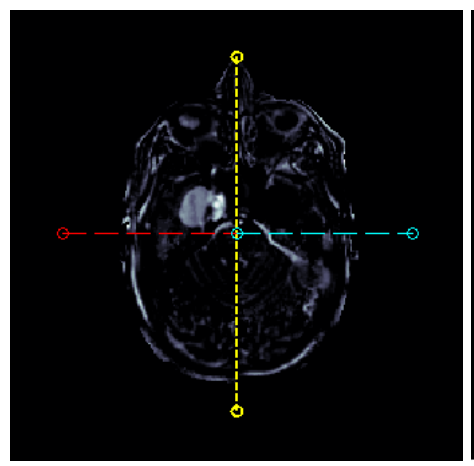

(b)

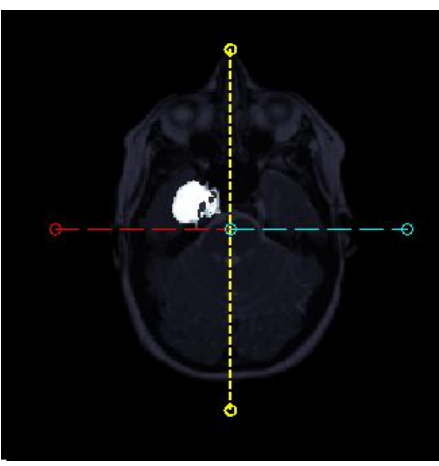

(c)

Fig.5. Human Tumor Segmentation (a) represents original input images; (b) are the re-oriented images with identified difference with respect to the symmetry axis; (c) are the final segmented region after region growing from the significant difference seeds derived from (b). 

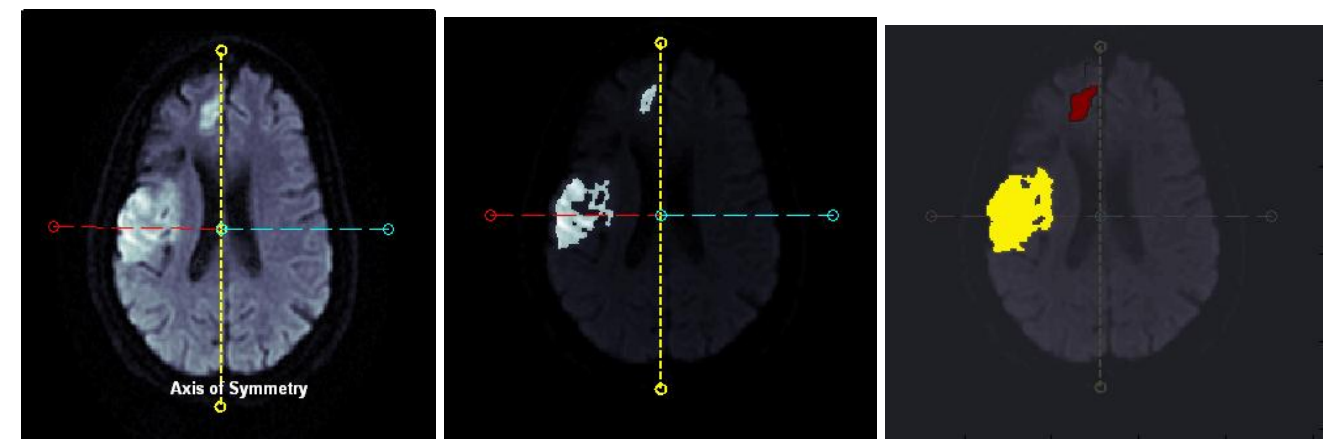

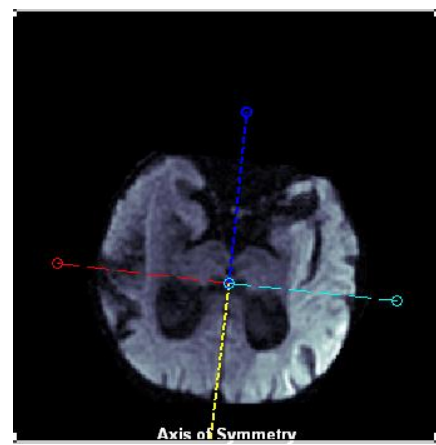

(a)

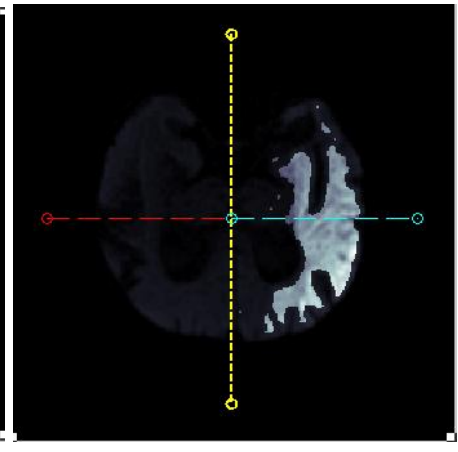

(b)

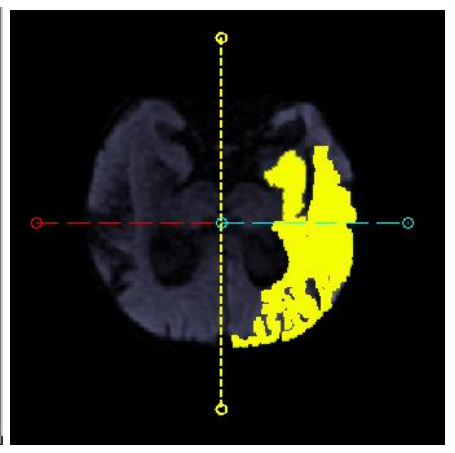

(c)

Fig.6. Human Stroke Segmentation in Diffusion Weighted Images.(DWI). (a) represents original input images; (b) are the re-oriented images with identified significant difference; (c) are the final segmented region after region growing.
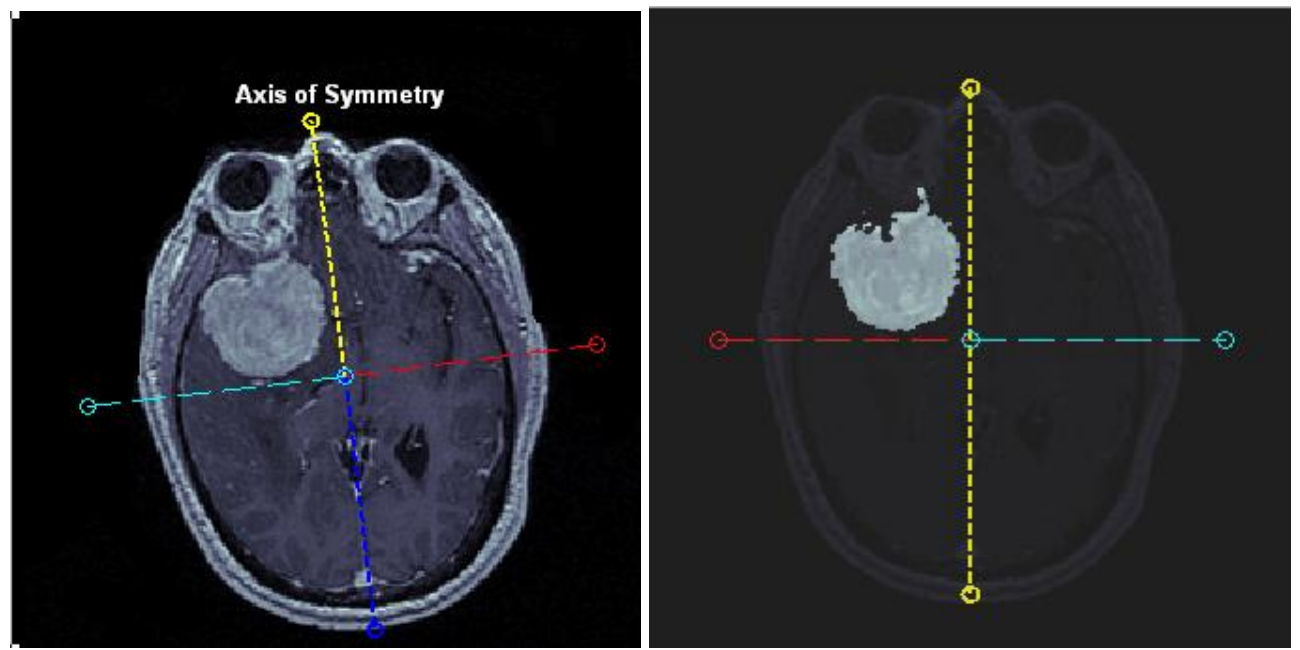

Fig.7. Human Tumor Segmentation 


\section{EVALUATION}

We have applied our automatic segmentation method to 10 rat stroke cases. The tool classifies the whole brain into healthy and pathological regions as shown in Figure 8. The surrogate of ground truth is derived from hand-segmented delineations from 3 human experts. The "experts" were trained technicians who showed noticeable intra and interoperator discrepancy.

$\begin{array}{llllllllll} & \text { S1 } & \text { S2 } & \text { S3 } & \text { S4 } & \text { S5 } & \text { S6 } & \text { S7 } & \text { S8 } & \text { S9 } \\ \text { GTA: } & 476.3 & & & & & & & & \\ \text { SA: } & 534 & 475 & 514 & 577 & 666 & 459 & 419 & 390 & 396 \\ \text { AD: } & 12.1 \% & 0.29 \% & 7.91 \% & 21.13 \% & 39.8 \% & 6.39 \% & 12.0 \% & 18.1 \% & 16.8 \% \\ \text { FNVF: } & 0.064 & 0.10 & 0.066 & 0.057 & 0.0 & 0.127 & 0.162 & 0.202 & 0.22 \\ \text { FPVF: } & 0.186 & 0.098 & 0.146 & 0.257 & 0.398 & 0.091 & 0.041 & 0.021 & 0.052 \\ \text { TPVF: } & 0.935 & 0.899 & 0.933 & 0.954 & 1 & 0.872 & 0.838 & 0.798 & 0.779 \\ \text { Figure of merit F: } & 0.533 & 0.669 & 0.620 & 0.509 & 0.558 & 0.656 & 0.803 & 0.852 & 0.865\end{array}$

Table 1. intra and inter-operator discrepancy when deriving the ground truth.
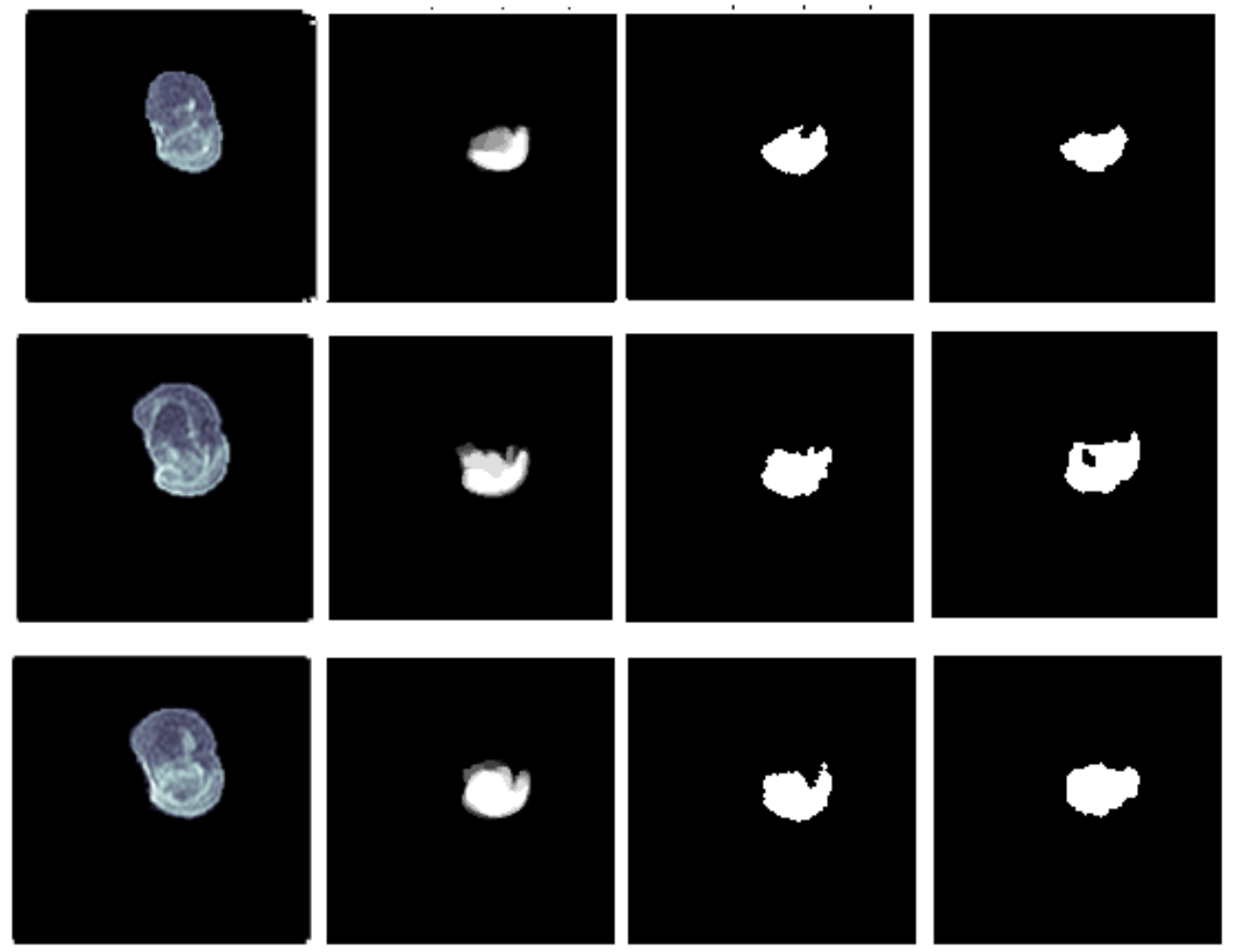

Fig.8. Validation of stroke segmentation on rat brain. The leftmost column is the original brain, second column is the surrogate ground truth, the third column contains the results of our proposed automated segmentation method, and the rightmost column is result of fuzzy connectedness segmentation. 


\begin{tabular}{|l|l|l|l|l|l|}
\hline & & Area Difference & $\begin{array}{l}\text { False Negative } \\
\text { Volume Fraction }\end{array}$ & $\begin{array}{l}\text { False Positive } \\
\text { Volume Fraction }\end{array}$ & $\begin{array}{l}\text { True Positive } \\
\text { Volume Fraction }\end{array}$ \\
\hline \multirow{2}{*}{ Scan1 } & RDM & $8.0292 \%$ & 0.0510949 & 0.131387 & 0.948905 \\
\cline { 2 - 6 } & Fuzzy & $11.1968 \%$ & 0.143569 & 0.255537 & 0.856431 \\
\hline \multirow{2}{*}{ Scan2 } & RDM & $0 \%$ & 0.114662 & 0.114662 & 0.885338 \\
\cline { 2 - 6 } & Fuzzy & $12.8404 \%$ & 0.0952236 & 0.223628 & 0.904776 \\
\hline \multirow{2}{*}{ Scan3 } & RDM & $3.42052 \%$ & 0.0704225 & 0.104628 & 0.929577 \\
\cline { 2 - 6 } & Fuzzy & $30.468 \%$ & 0.103076 & 0.407756 & 0.896924 \\
\hline
\end{tabular}

Table 2. Accuracy Measurement of 3 rat scans in figure 8 .

\section{CONCLUSIONS}

In this paper we present a generic method to compute axis of symmetry and quantification of asymmetry in brain imagery. There are various clinical applications that make use of brain imagery where quantification of asymmetry provides potential computer-assisted assessment and diagnostic tool: e.g. objective quantification of perfusion-weighted computed tomography in subarachnoid hemorrhage; quantification of previously undetected silent infarcts on MRperfusion in patient following carotid endarterectomy; pre-segmentation of ischemic stroke regions in a rat model of temporary middle cerebral artery occlusion; quantification of diffusion-weighted images and apparent diffusion coefficient maps in the detection of acute strokes. In each of these studies such a potential computer-assisted tool will have to be thoroughly validated in clinical setting using ROC (receiver operating characteristic) methodology [5], to show that the method improves diagnostic and/or assessment outcome. This approach consists of: 1) identifying the axis of symmetry; 2) measurements of pixel-wise symmetry between the two hemispheres; 3) statistical classification and modeling of the difference map; 4) evaluation of the system in assisted diagnosis. The preliminary results have shown that this approach has promise to achieve high precision and full automation in segmenting lesions in reflectional symmetry objects.

\section{References}

1. C. Imielinska, Y. Jin, X. Liu, at al., "Evaluation of ischemic stroke hybrid segmentation in a rat model of temporary middle cerebral artery occlusion using ground truth from histologic and MR data, SPIE2005, Medical Imaging.

2. X. Liu, C. Imielinska, J. Rosiene, at al.”A novel quantification method for determining previously undetected MR perfusion changes in patients with cognitive deficits following carotid endarterectomy", SPIE2005 Medical Imaging.

3. Imielinska C., X. Liu, M. Sughrue, E. Hagiwara, E.S. Connolly, A. D'Ambrosio, "Objective Quantification of Perfusion-Weighted Computer Tomography in the Setting of Acute Aneurysmal Subarachnoid Hemorrhage", Computer Assisted Radiology and Surgery, pp. 34-43, June 2004

4. C. Imielinska, X. Liu, J. Rosiene, at al, "Towards Objective Quantification of Perfusion- Weighted Computed Tomography in the Setting of Subarachnoid Hemorrhage: Quantifica tion of Symmetry and Automated Delineation of Vascular Territories". Academic Radiology.2005, 12:874-887.

5. C.E. Metz, "ROC methodology in radiologic imaging", Invest. Radiology, Vol. 21,pp. 720-733, 1986.

6. Michael R. Kaus, Simon K. Warfield, Arya Nabavi, Peter M. Black, Ferenc A. Jolesz, and Ron Kikinis Automated Segmentation of MR Images of Brain Radiology 2001; 218: 586.

7. D.Reisfeld, H.Wolfson and Y.Yeshurun. "Context Free Attentional operators: the generalized symmetry transform”. IJCV, vol.14,pp.119-130,1995. 
8. Gofman, Y.; Kiryati, N.; "Detecting symmetry in grey level images: the global optimization approach" Pattern Recognition, 1996., Proceedings of the 13th International Conference on, Volume: 1, 25-29 Aug. 1996

9. J.Bigun, "Recognition of Local Symmetries in Gray Value Images by Harmonic Functions", Proc. $9^{\text {th }}$ ICPR, pp.345-347, Rome, 1988.

10. J. Serra. Image Analysis and Mathematical Morphology. Academic Press, 1982.

11. L.P. Clarke, R.P. Velthuizen, S. Phuphanich, J.D. Schellenberg, J.A. Arrington, and M. Silbinger. MRI: Stability of Three Supervised Segmentation Techniques.

Magnetic Resonance Imaging, 11(1):95-106, 1993.

12. G. Gerig, J. Martin, R. Kikinis, O. Kübler, M. Shenton, and F.A. Jolesz. Unsupervised Tissue Type Segmentation of 3D dual-echo MR Head Data. Image and Vision Computing, 10(6):349-360, 1992.

IPMI 1991 Special Issue

13. R. Kikinis, M.E. Shenton, D.V. Iosifescu, R.W. McCarley, P. Saiviroonporn, H.H. Hokama, A. Robatino, D. Metcalf, C.G. Wible, C.M. Portas, R. Donnino, and F.A. Jolesz.

A Digital Brain Atlas for Surgical Planning, Model Driven Segmentation and Teaching. IEEE Transactions on Visualization and Computer Graphics, 2(3):232-241, 1996

14. B. Klaus, P. Horn. "Robot Vision", MIT press, pp. 48-58.

15. J. Rosiene, X. Liu, C. Imielinska, "Ray casting approach for boundary extraction and Fourier shape descriptor characterization", SPIE Electronic Imaging, Feb. 2005

16. Rafael C. Gonzalez, Richard E. Woods, "Digital Image Processing", Prentice Hall, $2^{\text {nd }}$ Edition, pp 613-614.2002.

17. Sylvain Prima, Sébastien Ourselin, and Nicholas Ayache, "Computation of the Mid-Sagittal Plane In 3-D Brain Images: IEEE transaction on medical imaging, vol.21, no.2, february 2002

18. B. A. Ardekani, J. Kershaw, M. Braun, and I. Kanno, "Automatic detection of the mid-sagittal plane in 3-D brain images," IEEE Trans. Med Imag., vol. 16, pp. 947-952, Dec. 1997.

19. D. Pham, C. Xu and J. Prince, "Current Methods in Medical Image Segmentation", Annual Review Biomedical Engineering, 2:315-337, 2000.

20. T. McInnerney and D. Terzopoulos. Deformable Models in Medical Image Analysis: A Survey. Medical Image Analysis, 1(2):91-108, 1996.

21. J. Udupa and S. Samarasekera, "Fuzzy Connectedness and Object Definition: Theory, Algorithms, and Applications in Image Segmentation." Graphical Models and Image Processing, vol. 58 (3), pp. 246-261, 1996.

22. C. Imielinska ., J. Udupa, D. Metaxas, Y. Jin, E. Angelini, T. Chen, Y Zhuge, "Hybrid Segmentation Methods", book chapter in "Insight into Images: Principles and Practice for Segmentation, Registration, and Image Analysis", edited by T. Yoo, A.K. Peters, March 2004

23. C. Imielinska, J. Rosiene, Y. Jin, et al.,"'Ground Truth for Evaluation of Ischemic Stroke Hybrid Segmentation in a Rat Model of Temporary Middle Cerebral Artery", Computer Assisted Radiology and Surgery, CARS 2005, 1281, pp 74-79. Berlin, June 2005.

24. P.K. Tulipano, W. Millar, C. Imielińska, , X. Liu, J. Rosiene, M. Sughrue, A. D'Ambrosio, "Quantification of Diffusion Weighted Images (DWI) and Apparent Diffusion Coefficiant Maps (ADC) in Detection of Acute Stroke", accepted to SPIE 2006 